\title{
SINGULAR INTEGRALS AND PARABOLIC EQUATIONS
}

BY B. FRANK JONES, JR.

Communicated by A. Zygmund, February 11, 1963

1. Introduction. A. P. Calderón and A. Zygmund $[1 ; 2]$ have studied a class of singular integrals, proving that such integrals generate continuous linear transformations of $L^{p}$ into $L^{p}, 1<p<\infty$. One of the many applications of their results is the derivation of integral estimates for derivatives of solutions of the Poisson equation $\Delta u=f$, where $\Delta=\partial^{2} / \partial x_{1}^{2}+\cdots+\partial^{2} / \partial x_{n}^{2}$. Corresponding results have been obtained for a different class of singular integrals; an application of these results is the derivation of integral estimates for derivatives of solutions of the parabolic equation $u_{t}-\Delta u=f$. We shall briefly outline the development of the singular integrals of Calderón and Zygmund as applied to the equation $\Delta u=f$, and then give the parallel development for the singular integrals associated with the equation $u_{t}-\Delta u=f$.

2. The equation $\Delta u=f$. In $n$-dimensional Euclidean space $R^{n}$ let $\Gamma(x)$ be the fundamental solution of Laplace's equation,

$$
\begin{array}{ll}
\Gamma(x)=-\frac{1}{2 \pi} \log \frac{1}{|x|}, & n=2, \\
\Gamma(x)=\frac{1}{(2-n) \omega_{n}}|x|^{2-n}, & n>2,
\end{array}
$$

where $|x|=\left(x_{1}^{2}+\cdots+x_{n}^{2}\right)^{1 / 2}$ and $\omega_{n}$ is the area of the sphere $|x|=1$. Let

$$
u(x)=\int_{R^{n}} \Gamma(x-y) f(y) d y
$$

where $f \in L^{p}\left(R^{n}\right)$. Then [1] the second partial derivatives of $u$ exist almost everywhere, and

$$
u_{x_{i} x_{j}}=\frac{1}{n} \delta_{i j} f(x)+\int_{R^{n}} k_{i j}(x-y) f(y) d y,
$$

where $\delta_{i i}=1, \delta_{i j}=0, i \neq j$, and

$$
k_{i j}(x)=\Gamma_{x_{i} x_{j}}=\frac{1}{\omega_{n}}|x|^{-n}\left(\delta_{i j}-n \frac{x_{i} x_{j}}{|x|^{2}}\right) .
$$

In particular, $\Delta u=f$. The kernel $k=k_{i j}$ has the properties that 


$$
\begin{aligned}
k(\alpha x) & =\alpha^{-n} k(x), \\
\int_{|x|=1} k(x) d \sigma & =0 .
\end{aligned}
$$$$
\alpha>0
$$

Now if $k(x)$ is any function satisfying (3) and (4), together with a certain mild smoothness or boundedness condition $[1 ; 2]$, then the mapping

$$
\rightarrow \int_{R^{n}} k(x-y) f(y) d y \equiv \lim _{\epsilon \rightarrow 0+} \int_{|x-y|>e} k(x-y) f(y) d y
$$

is a continuous transformation of $L^{p}$ into $L^{p}$, for $1<p<\infty$. In (2) the integral is also interpreted in the principal value sense of (5). The integral in (5) converges in $L^{p}$ and pointwise almost everywhere. In particular, the derivatives $u_{x_{i} x_{j}}$ in (2) satisfy

$$
\left\|u_{x_{i} x_{j}}\right\| L_{L^{p}} \leqq A_{p}\|f\|_{L^{p}}
$$

3. The equation $u_{t}-\Delta u=f$. For $0<t<\infty, x \in R^{n}$, let $\Gamma(x, t)$ be the fundamental solution of the heat equation $\left(u_{t}-\Delta u=0\right)$,

$$
\Gamma(x, t)=(4 \pi t)^{-n / 2} \exp \left(-\frac{|x|^{2}}{4 t}\right) .
$$

Let

$$
u(x, t)=\int_{0}^{t} \int_{R^{n}} \Gamma(x-y, t-s) f(y, s) d y d s,
$$

where $f \in L^{p}\left(R^{n} \times(0, \infty)\right)$. The analogue of $(2)$ is

$$
\begin{aligned}
u_{x_{i} x_{j}} & =\int_{0}^{t} \int_{R^{n}} k_{i j}(x-y, t-s) f(y, s) d y d s, \\
u_{t} & =f(x, t)+\int_{0}^{t} \int_{R^{n}} k^{\prime}(x-y, t-s) f(y, s) d y d s .
\end{aligned}
$$

Here

$$
\begin{aligned}
& k_{i j}(x, t)=\Gamma_{x_{i} x_{j}}=(4 \pi)^{-n / 2} t^{-n / 2-1}\left(-\frac{1}{2} \delta_{i j}+\frac{x_{i} x_{j}}{4 t}\right) \exp \left(-\frac{|x|^{2}}{4 t}\right) \\
& k^{\prime}(x, t)=\Gamma_{t}=(4 \pi)^{-n / 2} t^{-n / 2-1}\left(-\frac{1}{2} n+\frac{|x|^{2}}{4 t}\right) \exp \left(-\frac{|x|^{2}}{4 t}\right)
\end{aligned}
$$

In particular, $u_{t}-\Delta u=f$. 
Now let us consider kernels $k(x, t)$ satisfying

$$
\begin{aligned}
k(x, t) & =0, & & t<0, \\
k\left(\alpha x, \alpha^{2} t\right) & =\alpha^{-n-2} k(x, t), & & \alpha>0, \\
\int_{R^{n}} k(x, 1) d x & =0, & &
\end{aligned}
$$

together with certain mild smoothness and boundedness conditions. For instance, it is sufficient to require that $|k(x, 1)|+\left|k_{x_{i}}(x, 1)\right|$ $\leqq a e^{-b|x|}$ for some $a>0, b>0$. All these properties are satisfied by the kernels $k_{i j}(x, t)$ and $k^{\prime}(x, t)$. Note the analogy between conditions $\left(3^{\prime}\right),\left(4^{\prime}\right)$ and $(3),(4)$, respectively.

If $k(x, t)$ is any such function, then we shall consider the mapping

$$
\begin{aligned}
f & \rightarrow \int_{0}^{t} \int_{R^{n}} k(x-y, t-s) f(y, s) d y d s \\
& \equiv \lim _{\epsilon \rightarrow 0+} \int_{0}^{t-\epsilon} \int_{R^{n}} k(x-y, t-s) f(y, s) d y d s,
\end{aligned}
$$

the integral converging in $L^{p}$. The integrals in $\left(2^{\prime}\right)$ are also interpreted in this sense. Moreover the mapping $\left(5^{\prime}\right)$ defines a continuous transformation of $L^{p}$ into $L^{p}, 1<p<\infty$. Thus, for example, the derivatives $u_{x_{i} x_{j}}$ and $u_{t}$ in $\left(2^{\prime}\right)$ satisfy

$$
\left\|u_{x_{i} x_{j}}\right\|_{L^{p}}+\left\|u_{t}\right\|_{L^{p}} \leqq A_{p}\|f\|_{L^{p}}
$$

The precise statements and proofs of these and allied results will appear elsewhere. The proofs are similar to some of the arguments in $[1]$.

\section{REFERENCES}

1. A. P. Calderón and A. Zygmund, On the existence of certain singular integrals, Acta Math. 88 (1952), 85-139.

2. ——, On singular integrals, Amer. J. Math. 78 (1956), 289-309.

William Marsh Rice University 